Check for updates

Cite this: RSC Adv., 2017, 7, 48759

Received 25th August 2017

Accepted 9th October 2017

DOI: $10.1039 / c 7 r a 09430 b$

rsc.li/rsc-advances

\section{Revealing anisotropy and thickness dependence of Raman spectra for SnS flakes $\uparrow$}

\author{
Mingling Li, ${ }^{a}$ Yiming Wu, (D) a Taishen Li, ${ }^{a}$ Yulin Chen, (D) ${ }^{a}$ Huaiyi Ding, ${ }^{a}$ Yue Lin, ${ }^{a}$ \\ Nan Pan ${ }^{a b c}$ and Xiaoping Wang ${ }^{\star a b c}$
}

In this work, we have successfully synthesized SnS flakes with different thicknesses and systematically investigated their polarization-dependent Raman properties. It is found that the different Raman mode of SnS shows distinctly anisotropic thickness dependence. For $\mathrm{B}_{3 \mathrm{~g}}$ mode, the polar plot of Raman intensities is insensitive to the flake thickness. However, the behavior of $A_{g}$ mode is entirely different. Under parallel polarization configuration, with decreasing the flake thickness, the maximum Raman intensity of $A_{g}$ mode changes from the armchair direction to the zigzag direction with $514.5 \mathrm{~nm}$ excitation. The results can be understood by the complex Raman tensor owing to the large absorption of SnS. Moreover, under the perpendicular polarization configuration, the Raman intensity of $A_{g}$ mode along $45^{\circ}$ direction becomes apparently different from that along $135^{\circ}$ direction. Our finding not only deepens the understanding of anisotropic Raman properties of SnS but also provides inspiration for further studies on the other 2D IV-VI materials.

\section{Introduction}

$\mathrm{SnS}$ is an important member of the IV-VI two dimensional materials belonging to the space group $D_{2 \mathrm{~h}}^{16} \cdot{ }^{1-3}$ Like other $2 \mathrm{D}$ materials, the in-plane $S$ and $S n$ atoms are connected by covalent bonds, while the out-plane layers are connected by van der Waals interaction. ${ }^{\mathbf{1 , 4}}$ However, the existed electron lone pairs (i.e. $\mathrm{Sn}^{2+} 5 \mathrm{~s}^{2}$ ) can enhance the interlayer interaction and make it become a distorted $\mathrm{NaCl}$ type structure. ${ }^{5} \mathrm{SnS}$ is also an earthabundant, ${ }^{6}$ stable $^{7}$ and non-toxic ${ }^{8}$ material. Its large absorption coefficient $\left(\sim 10^{5} \mathrm{~cm}^{-1}\right),{ }^{9,10}$ as well as the predicted high carrier mobility, ${ }^{7}$ renders it a great of potential applications in optoelectronic and electronic devices. ${ }^{2,4,11-18}$ Moreover, similar to the black phosphorous (BP) with puckered honey-comb crystal structure, SnS also possesses anisotropic electronic, ${ }^{19,20}$ thermoelectric, ${ }^{21-23}$ piezoelectric ${ }^{24}$ and optical ${ }^{25,26}$ properties. Given the strongly anisotropic properties of $\mathrm{SnS}$, the $\mathrm{SnS}-\mathrm{SnS}_{x} \mathrm{Se}_{1-x}$ core-shell heterostructure with anisotropic photoresponse and

${ }^{a}$ Hefei National Laboratory for Physical Sciences at the Microscale, Department of Physics, University of Science and Technology of China, Hefei, Anhui 230026, P. R. China.E-mail: xpwang@ustc.edu.cn

${ }^{b}$ Synergetic Innovation Center of Quantum Information \& Quantum Physics, University of Science and Technology of China, Hefei, Anhui 230026, P. R. China

${ }^{c}$ Key Laboratory of Strongly-Coupled Quantum Matter Physics, Chinese Academy of Sciences, School of Physical Sciences, University of Science and Technology of China, Hefei, Anhui 230026, P. R. China

$\dagger$ Electronic supplementary information (ESI) available: AFM image of $13.2 \mathrm{~nm}$ SnS flake, polar plots of $\mathbf{B}_{3 \mathrm{~g}}\left(164.0 \mathrm{~cm}^{-1}\right)$ and $\mathbf{A}_{\mathbf{g}}\left(95.9 \mathrm{~cm}^{-1}\right.$ and $\left.192.0 \mathrm{~cm}^{-1}\right)$ with different thickness, the calculated polar plots of $\mathbf{A}_{\mathbf{g}}(\|)$ mode with different $|C| /|B|$ and $\varphi_{\mathrm{CB}}$, and variation of $\varphi_{\mathrm{CB}}$ with the thickness of SnS flake for $\mathbf{A}_{\mathbf{g}}$ mode $\left(192.0 \mathrm{~cm}^{-1}\right)$. See DOI: $10.1039 / \mathrm{c} 7 \mathrm{ra09430b}$ the SnS-based photodetector with highly anisotropic performance of near-infrared have been achieved. ${ }^{27,28}$ Additionally, the anisotropic photoresponse properties of SnS can be significantly improved by combining with the plasmon enhancement provided by various metallic nanoparticles. ${ }^{28-30}$ The anisotropic Raman property of SnS has also been observed and interpreted by Raman tensor theory. ${ }^{25}$ Like $\mathrm{BP},{ }^{31,32}$ the behavior can further be used to in situ identify the crystalline orientation to perform the anisotropic electrical and thermoelectrical measurement. ${ }^{20,25}$ However, similar to that observed in $\mathrm{BP},{ }^{33-38}$ the strong absorption of SnS would inevitably lead to complex elements in the Raman tensor and therefore change the anisotropic Raman properties with the thickness. However, the dependence of anisotropic behavior of SnS on the thickness has been overlooked. In this regard, it is interesting and imperative to investigate the anisotropic Raman properties of SnS flakes as well as its thickness dependence. However, to the best of our knowledge, there is no systematically study so far about this content, especially in-depth study on the complex Raman tensor elements.

In this work, we have successfully synthesized SnS flakes with different thicknesses by physical vapor deposition (PVD) method. We then systematically investigated the polarizationdependent Raman properties of SnS flakes through angleresolved polarized Raman spectroscopy (ARPRS). It is found that the different Raman modes of SnS show distinctly anisotropic dependence on the thickness of flake. Specially, for $\mathbf{B}_{\mathbf{3}}$ mode, the polar plots of Raman intensities for both parallel and perpendicular polarization configurations are insensitive to the flake thickness. On the contrary, the behavior of $\mathbf{A}_{\mathbf{g}}$ mode is 
entirely different. For the parallel polarization configuration, with decreasing the flake thickness, the maximum of Raman intensity changes from the armchair direction to the zigzag direction under $514.5 \mathrm{~nm}$ excitation. The result can be interpreted by introducing complex Raman tensor caused by the large absorption..$^{3-36,38}$ Furthermore, the Raman intensity along $45^{\circ}$ direction becomes different from that along $135^{\circ}$ direction under the perpendicular polarization configuration. Our finding enriches the understanding of fundamental anisotropic Raman properties of SnS and implies the necessity to consider the thickness of SnS flake to identify its crystalline orientation through Raman characterization.

\section{Experiments}

\section{Synthesis of 2D SnS flakes}

The SnS flakes with different thicknesses were synthesized by physical vapor deposition (PVD) method. ${ }^{25}$ The SnS powders ( $0.1 \mathrm{~g}$ Alfa Aesar 99.5\%) placed in the quartz tube were put onto the center of a horizontal furnace, and the freshly cleaved mica substrates were placed at $15-21 \mathrm{~cm}$ downstream. The furnace was evacuated to about $8 \mathrm{mTorr}$ and was then purged with ultrapure Ar. The temperature of the furnace center is set at $630-650{ }^{\circ} \mathrm{C}$. The pressure and the Ar flow were kept at $\sim 29$ Torr and $60 \mathrm{sccm}$ during the growth, respectively. The SnS vapor was carried by Ar flow and deposited onto the substrates, and the SnS flakes were formed subsequently. The growth was maintained for 5-15 min. Then the furnace was cooled down immediately by opening the furnace.

\section{Transfer method}

The SnS flakes were transferred from mica to $\mathrm{SiO}_{2} / \mathrm{Si}$ substrate by a water ultrasonic method. The flakes on mica were spincoated with PMMA (A4 495) at $3000 \mathrm{rpm}$ for $45 \mathrm{~s}$, and then were put onto a hot plate and kept at $120{ }^{\circ} \mathrm{C}$ for $3 \mathrm{~min}$. The PMMA film were separated from the mica substrate through dipping it into water and by ultrasonic for several minutes. After that, PMMA containing flakes was transferred onto $\mathrm{SiO}_{2} / \mathrm{Si}$ substrate and gently dried with lamp heating. Finally, PMMA was removed by dipping in ethyl acetate and acetone, respectively.

\section{TEM characterization}

TEM, HRTEM images and SAED patterns were collected by a FEI Talos transmission electron microscope operated at $200 \mathrm{kV}$. The samples for characterization were transferred onto copper grids by the same method described above.

\section{Raman measurement}

The Raman spectra were obtained by LabRAM HR 800 Raman system under $514.5 \mathrm{~nm}$ and $632.8 \mathrm{~nm}$ excitation, respectively. The intensities on the samples for both $514.5 \mathrm{~nm}$ and $632.8 \mathrm{~nm}$ laser in our experiment are controlled at about $0.5 \mathrm{~mW}(0.4$ $\mathrm{mW}$ ) without (with) a polarizer in the exciting light path. We used $100 \times$ objective lens (N.A. $=0.9$ ) and 600 lines per $\mathrm{mm}$ grating to collect Raman signals. The spectrum resolutions for
$514.5 \mathrm{~nm}$ and $632.8 \mathrm{~nm}$ excitation are $1.88 \mathrm{~cm}^{-1}$ and $1.21 \mathrm{~cm}^{-1}$, respectively. In polarization-dependent Raman measurement, a polarizer was placed in the incident light path to improve the polarization of exciting light and weaken the depolarization effects caused by objective lens. The direction of polarizer is fixed and the direction of analyzer is alterable. Two configurations of parallel $(\|)$ and perpendicular $(\perp)$ polarization were adopted in the measurement, in which the polarization direction of the polarizer and the analyzer were kept parallel or perpendicular to each other, respectively. The polarization direction of exciting laser with respect to the sample was changed by rotating a custom sample stage by every 5/10/15 degrees. The position and spot area of excitation were cautiously kept the same for each measurement.

\section{Results and discussion}

Fig. 1a shows the optical image of a typical result of as-grown SnS flakes. As seen, all flakes have square-like morphologies and their averaged size can reach as large as $10 \mu \mathrm{m}$, which can be observed more clearly in Fig. 1b. Atomic force microscopy (AFM) (Seiko Instrument Industry Co.) characterization demonstrates that the flake has a smooth surface morphology and its thickness can be down to $7.6 \mathrm{~nm}$, as seen in Fig. 1c. The bright particles on the flake surface are the residues from the transfer process (see Fig. S1 in $\mathrm{ESI}_{\dagger}^{\dagger}$ ). We also note that the morphology of $7.6 \mathrm{~nm}$ SnS flake shows rounded corners, while most of flakes shown in Fig. 1a demonstrate sharp corners. Considering that the perfect rhombus structure with sharp
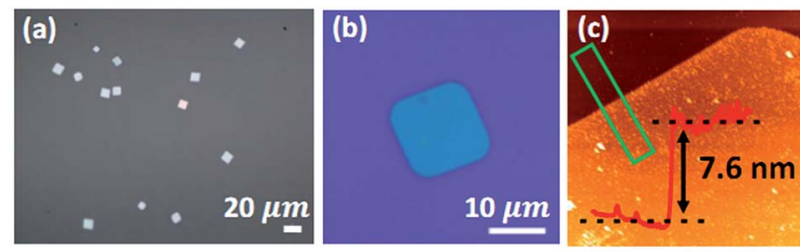

(d)
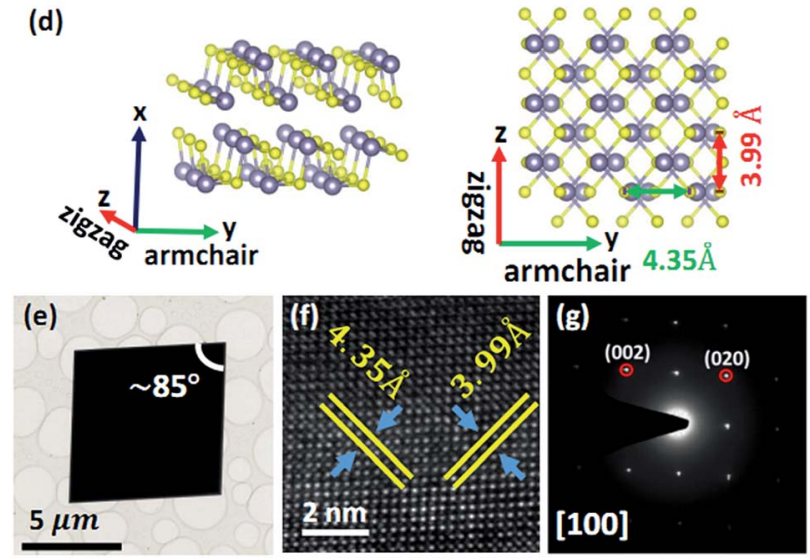

Fig. 1 (a) Optical image of as-grown SnS flakes on mica substrate and (b) a thin $\mathrm{SnS}$ flake transferred onto $\mathrm{SiO}_{2} / \mathrm{Si}$ substrate. (c) AFM image of $7.6 \mathrm{~nm}$ thick SnS flake. (d) Schematic of crystal structure of SnS from side view (left) and top view (right). (e) TEM image of a SnS flake with a corner angle of $\sim 85^{\circ}$. (f) HRTEM image and (g) SAED pattern of SnS flake. 
corners is the thermodynamic equilibrium state of SnS flake ${ }^{20}$ and the growth time for the thin flakes is much short $(<5 \mathrm{~min})$, we think that there might be no enough time to reach the thermodynamic equilibrium for the thin flakes, resulting in their rounded corners morphology. Additionally, we found that it is difficult to obtain the very thin flakes, because SnS has a relatively strong interlayer interaction ${ }^{5,39}$ and the complicated phases of S and Sn compounds. ${ }^{40}$

We carried out transmission electron microscopy (TEM) as well as the selected area electron diffraction (SAED) characterizations to investigate the structure of the SnS flake and evaluate its crystal quality. Fig. 1d depicts the schematic of crystal structure of SnS. Similar to BP, SnS has also a puckered honeycomb structure, and the two in-plane principle axes are corresponding to armchair direction ( $y$ axis) and zigzag direction ( $z$ axis), respectively. As seen in Fig. 1e, the SnS flake transferred onto the TEM grids shows a rhombus shape, and the corner angle is estimated to $\sim 85^{\circ}$, which is in good agreement with previous reports. ${ }^{20,25}$ Additionally, the perfect 2D rhombus lattice can be found in the high resolution TEM image taken from the flake (Fig. 1f). Two lattice spacings corresponding to the (010) and (001) planes are obtained to be about $4.35 \AA$ and $3.99 \AA$, respectively. The values are well consistent with the previous results. ${ }^{20,25,41}$ Fig. $1 \mathrm{~g}$ demonstrates the corresponding SAED pattern along [100] zone axis of the flake, and the well-defined diffraction spots reveal the good crystalline of as-grown $\mathrm{SnS}$ flake. It has been pointed out that the crystalline direction of the SnS can be readily identified from the rhombus shape, i.e., the longer (shorter) diagonal direction is corresponding to the armchair (zigzag) direction. ${ }^{20,25}$ Therefore, the method is used to ascertain the armchair (or zigzag) direction of the flake for the anisotropic characterization of Raman spectroscopy in the following paragraphs.

The flakes were characterized by the Raman spectroscopy with $514.5 \mathrm{~nm}$ laser and the spectra of flakes with different thicknesses are shown in Fig. 2a. As seen, for the thick flakes (169.0 $\mathrm{nm}$ and $229 \mathrm{~nm}$ ), six peaks located at $40.2 \mathrm{~cm}^{-1}$, $49.1 \mathrm{~cm}^{-1}, 95.9 \mathrm{~cm}^{-1}, 164.0 \mathrm{~cm}^{-1}, 192.0 \mathrm{~cm}^{-1}$ and $219.5 \mathrm{~cm}^{-1}$

(a)

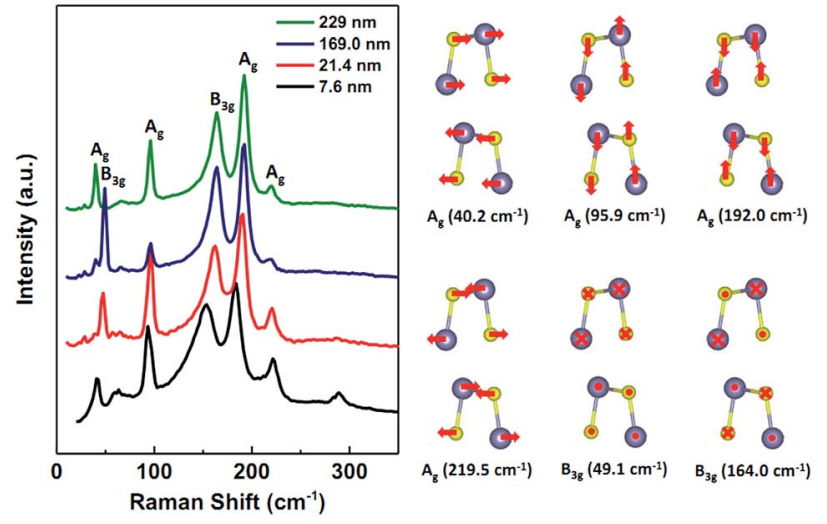

Fig. 2 (a) Raman spectra of SnS flakes with different thicknesses under $514.5 \mathrm{~nm}$ laser excitation. (b) Vibration diagrams for $A_{g}$ modes and $B_{3 g}$ modes. can be observed clearly. (The Raman peak positions are obtained from fitting the spectrum and its uncertainty is about $0.3 \mathrm{~cm}^{-1}$ ). These peaks can be attributed to the different Raman modes of $\mathbf{A}_{\mathbf{g}}$ and $\mathbf{B}_{\mathbf{3}}$ of SnS, respectively, ${ }^{41}$ as shown in the schematic of Fig. 2b. Specifically, the low frequency $\mathbf{A}_{\mathbf{g}}$ $\left(40.2 \mathrm{~cm}^{-1}\right)$ and high frequency $\mathbf{A}_{\mathbf{g}}\left(219.5 \mathrm{~cm}^{-1}\right)$ are corresponding to the layer shear mode and the "NaCl" type vibration, respectively. Two other $\mathbf{A}_{\mathbf{g}}$ peaks $\left(95.9 \mathrm{~cm}^{-1}, 192.0 \mathrm{~cm}^{-1}\right)$ are originated from the "waving" and the "breathing" modes, while $\mathbf{B}_{3 \mathrm{~g}}\left(49.1 \mathrm{~cm}^{-1}\right)$ and $\mathbf{B}_{3 \mathrm{~g}}\left(164.0 \mathrm{~cm}^{-1}\right)$ can be attributed to the shear vibration and the "NaCl" type vibration along zigzag direction, respectively. ${ }^{41}$ From Fig. $2 \mathrm{a}$, a little red shift of Raman peaks can be observed with decreasing the flake thickness. Taking $7.6 \mathrm{~nm}$ thick SnS as an example, the positions of three strong peaks are located at about $93.9 \mathrm{~cm}^{-1}\left(\mathbf{A}_{\mathrm{g}}^{1}\right), 151.7 \mathrm{~cm}^{-1}$ $\left(\mathbf{B}_{3 \mathrm{~g}}\right)$ and $183.2 \mathrm{~cm}^{-1}\left(\mathbf{A}_{\mathbf{g}}^{2}\right)$, respectively. Compared to the results of $229 \mathrm{~nm}$ flake, the red shifts can be estimated about $2.0 \mathrm{~cm}^{-1}$ ( $\mathbf{A}_{\mathrm{g}}^{\mathbf{1}}$ mode), $12.3 \mathrm{~cm}^{-1}$ ( $\mathbf{B}_{3 \mathrm{~g}}$ mode) and $8.8 \mathrm{~cm}^{-1}$ ( $\mathbf{A}_{\mathrm{g}}^{2}$ mode), respectively. The phenomenon might be ascribed to the change of bond angle in the thin flake, leading to the smaller of the restoring force for the vibration and the red shift of the corresponding Raman peak. ${ }^{7}$

To further investigate the anisotropic Raman response of $\mathrm{SnS}$, the polarization-dependent Raman spectroscopy was performed on the SnS flake. As seen in Fig. 3a, the Raman spectrum was collected through the back-scattering configuration with a polarizer and an analyzer placed in the incident and the scattering light paths, respectively. Two configurations of parallel $(\|)$ and perpendicular $(\perp)$ polarization were adopted in the measurement, in which the polarization direction of the polarizer and the analyzer were kept parallel or perpendicular to each other, respectively (Fig. 3a). The polarization angle, defined as the angle between the polarization direction of incident light and the armchair direction of SnS flake, can be tuned from $0^{\circ}$ to $360^{\circ}$ through rotating a custom sample stage.

Fig. $3 \mathrm{~b}$ and $\mathrm{c}$ demonstrate the anisotropic Raman response of $229 \mathrm{~nm}$ SnS flake to the different polarization angles with $514.5 \mathrm{~nm}$ excitation under parallel and perpendicular polarization configurations, respectively. As shown in Fig. $3 \mathrm{~b}, \mathbf{A}_{\mathbf{g}}$ $\left(95.9 \mathrm{~cm}^{-1}\right), \mathbf{B}_{3 \mathbf{g}}\left(164.0 \mathrm{~cm}^{-1}\right)$ and $\mathbf{A}_{\mathbf{g}}\left(192.0 \mathrm{~cm}^{-1}\right)$ are three strong peaks among the Raman spectra, we therefore concentrate on these three peaks in the following investigation. It can be seen from Fig. $3 \mathrm{~b}$ and $\mathrm{c}$ that the peak intensities of $\mathbf{A}_{\mathbf{g}}$ and $\mathbf{B}_{3 \mathrm{~g}}$ change periodically with the polarization angle, indicating the anisotropic Raman signals of the SnS flake. The behavior can be observed more clearly with the polar plots for the modes of $\mathbf{A}_{\mathbf{g}}$ $\left(192.0 \mathrm{~cm}^{-1}\right)$ and $\mathbf{B}_{3 \mathrm{~g}}\left(164.0 \mathrm{~cm}^{-1}\right)$, as shown in Fig. $3 \mathrm{~d}-\mathrm{g}$. As seen in Fig. 3d, the Raman intensity of $\mathbf{A}_{\mathbf{g}}\left(192.0 \mathrm{~cm}^{-1}, \|\right)$ along armchair is larger than that along zigzag direction. However, it is worth noting that the intensity of zigzag direction is the secondary maximum here, rather than the minimum as the previous works reported. ${ }^{20,25}$ This implies that the complex Raman tensor should be considered to interpret the result as that treated for $\mathrm{BP}^{33-36,38}$ On the other hand, as for the modes of $\mathbf{B}_{3 \mathrm{~g}}\left(164.0 \mathrm{~cm}^{-1}, \|\right)$, the intensities along armchair direction and zigzag direction are smallest while the intensities along $45^{\circ}$ $\left(225^{\circ}\right)$ and $135^{\circ}\left(315^{\circ}\right)$ are the largest (Fig. 3f). 
(a)

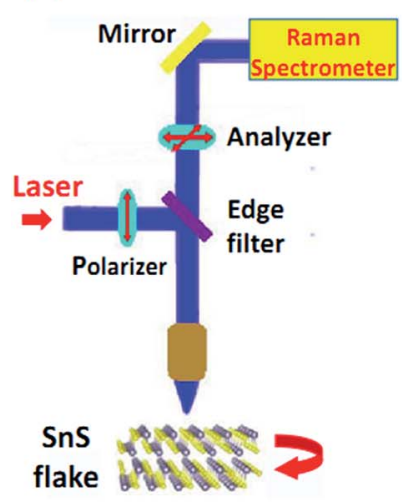

(b) II

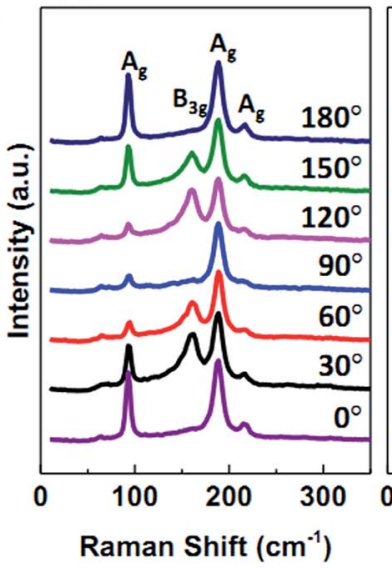

(c) $\perp$

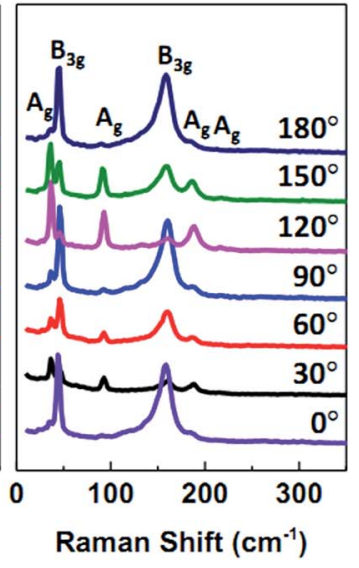

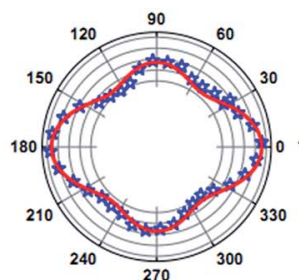

(d) $A_{g} \|\left(192.0 \mathrm{~cm}^{-1}\right)$

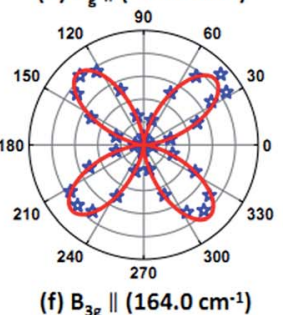

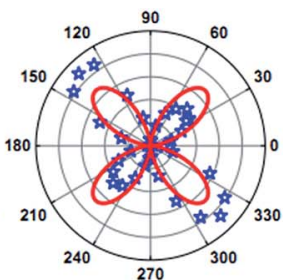

(e) $A_{g} \perp\left(192.0 \mathrm{~cm}^{-1}\right)$

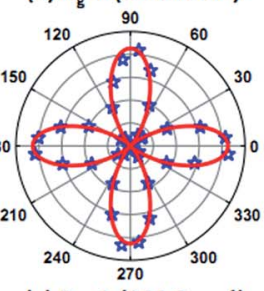

(g) $B_{3 \mathrm{~g}} \perp\left(164.0 \mathrm{~cm}^{-1}\right)$

Fig. 3 (a) Schematic diagram of Raman measurement for SnS flake. Polarization-dependent Raman spectra of $229 \mathrm{~nm}$ thick flake with $514.5 \mathrm{~nm}$ laser excitation under (b) parallel and (c) perpendicular polarization configurations. (d-g) The polar plots of intensity of $A_{g}\left(192 \mathrm{~cm}^{-1}\right)$ and $B_{3 g}$ $\left(164.0 \mathrm{~cm}^{-1}\right)$ under parallel and perpendicular polarization, respectively.

Theoretically, the Raman intensity can be calculated by $I \propto$ $\left|\mathbf{e}_{\mathbf{i}} \cdot \mathbf{R} \cdot \mathbf{e}_{\mathbf{s}}\right|^{2}$ and the anisotropic behavior is ascribed to the Raman tensor $\mathbf{R}$, which is determined by the detailed crystal structure of material. Owing to its large absorption coefficient, ${ }^{9,10}$ the Raman tensor elements of the SnS should be complex. Consequently, similar to the treatment for $\mathrm{BP}^{33-36,38}$ the Raman tensors for $\mathbf{A}_{\mathbf{g}}$ and $\mathbf{B}_{3 \mathbf{g}}$ of SnS can be described by

$$
\begin{aligned}
\mathbf{R}\left(\mathbf{A}_{\mathbf{g}}\right) & =\left(\begin{array}{ccc}
|\mathbf{A}| \mathbf{e}^{\mathbf{i} \varphi_{\mathcal{A}}} & 0 & 0 \\
0 & |\mathbf{B}| \mathbf{e}^{\mathbf{i} \varphi_{\mathbf{B}}} & 0 \\
0 & 0 & |\mathbf{C}| \mathbf{e}^{\mathbf{i} \varphi_{\mathrm{C}}}
\end{array}\right) \\
\mathbf{R}\left(\mathbf{B}_{3 \mathbf{g}}\right) & =\left(\begin{array}{ccc}
0 & 0 & 0 \\
0 & 0 & |\mathbf{F}| \mathbf{e}^{\mathbf{i} \varphi_{\mathrm{F}}} \\
0 & |\mathbf{F}| \mathbf{e}^{\mathbf{i} \varphi_{\mathrm{F}}} & 0
\end{array}\right)
\end{aligned}
$$

For the polarization angle of $\theta$, the incident light vector is $\mathbf{e}_{\mathbf{i}}$ $=(0, \cos \theta, \sin \theta)$, while the scattering light vector is $\mathbf{e}_{\mathbf{s}}=(0$, $\cos \theta, \sin \theta)$ for parallel polarization but $\mathbf{e}_{\mathbf{s}}=(0,-\sin \theta, \cos \theta)$ for the perpendicular case. Hence, the anisotropic Raman intensities for different modes can be expressed as:

$I\left(\mathbf{A}_{\mathbf{g}}, \|\right) \propto|B|^{2} \cos ^{4} \theta+|C|^{2} \sin ^{4} \theta+2|B \| C| \cos ^{2} \theta \sin ^{2} \theta \cos \varphi_{\mathrm{CB}}$

$$
\begin{gathered}
I\left(\mathbf{B}_{\mathbf{3 g}}, \|\right) \propto|F|^{2} \sin ^{2} 2 \theta \\
I\left(\mathbf{A}_{\mathbf{g}}, \perp\right) \propto \frac{|B|^{2}+|C|^{2}-2|B||C| \cos \varphi_{\mathrm{CB}}}{4} \sin ^{2} 2 \theta \\
I\left(\mathbf{B}_{\mathbf{3 g}}, \perp\right) \propto|F|^{2} \cos ^{2} 2 \theta
\end{gathered}
$$

where $\varphi_{\mathrm{CB}}=\left|\varphi_{\mathrm{C}}-\varphi_{\mathrm{B}}\right|$ is the phase difference between Raman tensor elements $|\mathbf{B}| \mathbf{e}^{\mathbf{i} \varphi_{\mathbf{B}}}$ and $|\mathbf{C}| \mathbf{e}^{\mathbf{i} \varphi_{\mathrm{C}}}$.

We fitted the experiment data with above equations, and the results are plotted in Fig. $3 d-g$ with red solid lines. As seen, the fitting is well consistent with the experiment results. Moreover, combined with the theory and experiment setup, the anisotropic behavior of $\mathbf{A}_{\mathbf{g}}\left(192.0 \mathrm{~cm}^{-1}\right)$ mode can be understood from the fact that its vibration motion has more component in armchair direction than that in zigzag direction. ${ }^{33-36,38}$ As for the mode of $\mathbf{B}_{3 \mathrm{~g}}\left(164.0 \mathrm{~cm}^{-1}\right)$, there only exist off-diagonally nonzero elements in the Raman tensor. Therefore, when the polarization of incident light is along armchair (zigzag) direction, the polarization of Raman scattering light will switch to zigzag (armchair) direction. This leads to the intensities of $\mathbf{B}_{\mathbf{3}} \mathbf{g}$ $\left(164.0 \mathrm{~cm}^{-1}\right)$ along armchair and zigzag directions become zero under the parallel polarization configuration (Fig. 3f) but achieve the maximum under the perpendicular polarization configuration, as shown in Fig. 3g.

In order to know whether the anisotropic Raman properties are dependent on the flake thickness, we carried out polarization-dependent Raman measurements on the SnS flakes with different thicknesses. Fig. $4 \mathrm{a}$ and $\mathrm{b}$ show the results for $\mathbf{B}_{3 \mathrm{~g}}\left(164.0 \mathrm{~cm}^{-1}\right)$ under parallel and perpendicular polarization configurations, respectively. As seen, for both configurations, the polar plots of Raman intensity for different thick SnS flakes are similar to those shown in Fig. $3 \mathrm{f}$ and $\mathrm{g}$, indicating that the $\mathbf{B}_{\mathbf{3 g}}$ mode is insensitive to the flake thickness. Similar results with $632.8 \mathrm{~nm}$ excitation are presented in Fig. S2. $\dagger$ The behavior can be easily understood by the description of eqn (2) and (4). However, as compared to $\mathbf{B}_{3 \mathbf{g}}$ mode, the situation of $\mathbf{A}_{\mathbf{g}}$ mode is distinctly different. As seen in the Fig. $4 \mathrm{c}$ for the mode of $\mathbf{A}_{\mathbf{g}}\left(192.0 \mathrm{~cm}^{-1}, \|\right)$, the Raman intensity along the armchair direction is larger than that along the zigzag direction for the thick flakes, but the behavior reverses for the thin flake, i.e., the Raman intensity along the zigzag direction becomes larger. This unusual result indicates that the anisotropic Raman response of SnS is strongly dependent on the flake thickness.

As indicated in the aforementioned paragraph, the intensity of $\mathbf{A}_{\mathbf{g}}$ mode under parallel polarization configuration can be described by eqn (1), from which we can find that the polar plot of Raman intensity is determined by three parameters, $|C|,|B|$ 


\begin{tabular}{|c|c|c|c|c|}
\hline & $7.6 \mathrm{~nm}$ & $16.1 \mathrm{~nm}$ & $29.0 \mathrm{~nm}$ & $229.0 \mathrm{~nm}$ \\
\hline \multirow{2}{*}{\multicolumn{5}{|c|}{$\begin{array}{l}\text { (a) } \\
B_{3 g^{\prime}} \| \\
\left(164.0 \mathrm{~cm}^{-1}\right)\end{array}$}} \\
\hline & & & & \\
\hline \multicolumn{5}{|l|}{ (b) } \\
\hline \multicolumn{5}{|l|}{$\begin{array}{c}B_{3 g^{\prime}} \perp \\
\left(164.0 \mathrm{~cm}^{-1}\right)\end{array}$} \\
\hline \multicolumn{5}{|l|}{ (c) } \\
\hline \multirow{2}{*}{\multicolumn{5}{|c|}{$\begin{array}{l}\text { (d) } \\
A_{g^{\prime}} \perp \\
\left(192.0 \mathrm{~cm}^{-1}\right)\end{array}$}} \\
\hline & & & & \\
\hline & & & & \\
\hline
\end{tabular}

Fig. 4 Polar plots of Raman intensities of $B_{3 g}\left(164.0 \mathrm{~cm}^{-1}\right)$ and $A_{g}$ $\left(192.0 \mathrm{~cm}^{-1}\right)$ for different thick SnS flakes with $514.5 \mathrm{~nm}$ laser excitation under parallel and perpendicular polarization configurations, respectively. The red curves are fitting results.

and $\varphi_{\mathrm{CB}}$. Several calculated results based on eqn (1) are shown in Fig. S3. $\dagger$ Obviously, $|C|^{2} /|B|^{2}$ is equal to the intensity ratio of the zigzag direction to the armchair direction, reflecting the Raman anisotropy in the $y-z$ plane, while $\varphi_{\mathrm{CB}}$ determines the concave degree of the intensity pattern along nearby $45^{\circ}$ (or $225^{\circ}$ ) and $135^{\circ}$ (or $315^{\circ}$ ). Compared to the calculated pattern shown in Fig. $\mathrm{S} 3, \dagger$ the result in Fig. $4 \mathrm{c}$ therefore demonstrates that the ratio of $|C|^{2} /|B|^{2}$ varies with the thickness of SnS flake. The finding is important because it suggests that it is impossible to identify the armchair direction of SnS flake only through the maximum of Raman intensity, as that used previously. ${ }^{20,25}$ Moreover, this variation tendency that the Raman intensity along zigzag direction increases with decreasing the flake thickness can also be observed with $632.8 \mathrm{~nm}$ excitation (Fig. S4d†).

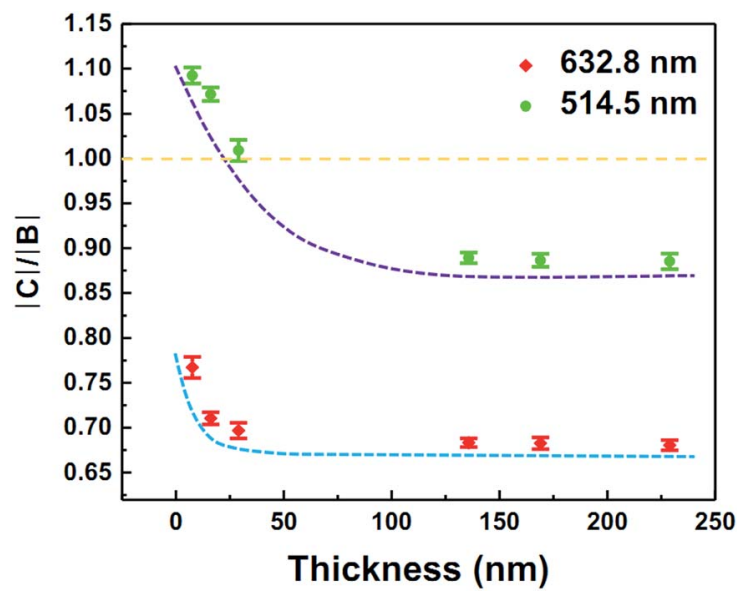

Fig. 5 Variation of $|C| /|B|$ with the thickness of $S n S$ flake for $A_{g}$ mode $\left(192.0 \mathrm{~cm}^{-1}\right)$ with $514.5 \mathrm{~nm}$ and $632.8 \mathrm{~nm}$ laser excitation, respectively. The lines are guides for eye.
We used eqn (1) to fit the results of Fig. $4 \mathrm{c}$ and $\mathrm{S} 4 \mathrm{~d}, \dagger$ and the fitting parameters $|C| /|B|$ and $\varphi_{\mathrm{CB}}$ are summarized in Fig. 5 and $\mathrm{S} 5, \uparrow$ respectively. As seen in Fig. 5, two features can be found clearly. Firstly, for both $514.5 \mathrm{~nm}$ and $632.8 \mathrm{~nm}$ excitations, the value of $|C| /|B|$ decreases quickly with increasing the flake thickness and then reaches steady value for fairly thick flakes. The variation of $\varphi_{\mathrm{CB}}$ on the flake thickness has similar tendency (Fig. S5†). According to the semi-classical Raman theory, Raman tensor elements $|\mathbf{B}| \mathbf{e}^{\mathbf{i} \varphi_{\mathbf{B}}}$ and $|\mathbf{C}| \mathbf{e}^{\mathbf{i} \varphi_{\mathbf{C}}}$ are the partial derivatives of the dielectric constant of SnS with respect to the normal coordinates of the corresponding phonon modes along $y y$ and $z z$ directions, respectively. ${ }^{33,38,42}$ In this context, we consider that the variation of $|C| /|B|$ with the flake thickness is probably attributed to the change of dielectric property for thin SnS flake as compared to that of the bulk. More importantly, Fig. 5 demonstrates $|C|>$ $|B|$ for thin SnS flakes under $514.5 \mathrm{~nm}$ excitation, which is distinctly unusual as compared to the previous reports. ${ }^{20,25} \mathrm{We}$ ascribe the underlying reason to the strongly resonant absorption in zigzag direction for thin flake, ${ }^{43}$ leading to the change of the dielectric dispersion relationship and hence the Raman tensor. More investigation is surely needed to elucidate this conjecture in the future work. Secondly, the values of $|C| /|B|$ with $514.5 \mathrm{~nm}$ excitation are larger than those with $632.8 \mathrm{~nm}$ excitation for all different thick flakes. The behavior can be further observed clearly by comparing Fig. $4 \mathrm{c}$ and $\mathrm{S} 4 \mathrm{~d}$. $\dagger$ Because $|C| /|B|=1$ is the indicative of Raman isotropy along armchair and zigzag directions, the result in Fig. 5 suggests that the anisotropic Raman spectrum can be observed more readily with $632.8 \mathrm{~nm}$ excitation.

Finally, we return to the anisotropic properties of $\mathbf{A}_{\mathbf{g}}$ $\left(192.0 \mathrm{~cm}^{-1}, \perp\right)$ of SnS flakes with different thicknesses. As seen in Fig. 4d, all polar plots show the maximum values of Raman intensities along $45^{\circ}$ (or $225^{\circ}$ ) and $135^{\circ}$ (or $315^{\circ}$ ), while the minimum intensities are along armchair and zigzag directions. This is ascribed to that all non-diagonal elements in the Raman tensor of $\mathbf{A}_{\mathbf{g}}$ modes are zero. As a consequence, when the polarization of excitation light is along armchair or zigzag direction, the final intensity of Raman scattering will become zero under perpendicular polarization configuration. Also from the prediction of eqn (2), the Raman intensity along $45^{\circ}$ (or $225^{\circ}$ ) direction must be equal to that along $135^{\circ}$ (or $315^{\circ}$ ) for $\mathbf{A}_{\mathbf{g}}(\perp$ ) mode. However, this contrasts obviously to the experimental observation for $\mathbf{A}_{\mathbf{g}}$ mode (192.0 $\mathrm{cm}^{-1}, \perp$ ) shown in Fig. $4 \mathrm{~d}$, in which the intensity difference between $45^{\circ}\left(225^{\circ}\right)$ and $135^{\circ}\left(315^{\circ}\right)$ is apparent for the SnS flakes, especially for $29.0 \mathrm{~nm}$ and $229 \mathrm{~nm}$ flakes. The behavior has also been reported in $\mathrm{SnS} .{ }^{25}$ It is difficult to understand this phenomenon at present due to the fact that the $45^{\circ}$ (or $225^{\circ}$ ) and $135^{\circ}$ (or $315^{\circ}$ ) directions are equal from the viewpoint of crystal structure. One possible reason is attributed to the circular dichroism originated from the uniquely puckered anisotropic structure of SnS, leading to the different absorption along $45^{\circ}$ and $135^{\circ}$ directions.

\section{Conclusions}

We have successfully synthesized SnS flakes with different thicknesses by physical vapor deposition method. The anisotropy and the thickness dependence of Raman properties are 
systematically investigated through the polarization-dependent Raman spectra. Both $\mathbf{B}_{\mathbf{3 g}}$ and $\mathbf{A}_{\mathbf{g}}$ modes can be observed from the SnS flakes but they show distinctly anisotropic thickness dependence. On the one hand, the anisotropy of $\mathbf{B}_{\mathbf{3}}$ mode is found to be independent of the flake thickness. On the other hand, the $\mathbf{A}_{\mathbf{g}}$ mode shows entirely different behavior. Under parallel polarization configuration and $514.5 \mathrm{~nm}$ excitation, the maximum Raman intensity of $\mathbf{A}_{\mathbf{g}}$ mode changes from the armchair direction to the zigzag direction with decreasing the flake thickness. The result is interpreted through the complex Raman tensor due to the large absorption of SnS. Under the perpendicular polarization configuration, the Raman intensity of $\mathbf{A}_{\mathbf{g}}$ mode along $45^{\circ}$ direction becomes different from that along $135^{\circ}$ direction. Our finding also implies that the anisotropic electron-phonon interactions in SnS flakes are dependent on the thickness. This work can enrich the understanding of fundamental anisotropic Raman properties of SnS and provide the inspiration for further studies on other $2 \mathrm{D}$ anisotropic materials.

\section{Conflicts of interest}

There are no conflicts of interest to declare.

\section{Acknowledgements}

We acknowledge the financial supports from MOST of China (2016YFA0200602), National Natural Science Foundation of China (21421063, 11474260, 11374274, 11504364), the Chinese Academy of Sciences (XDB01020200) and Anhui Natural Science Foundation (1608085QA17).

\section{Notes and references}

1 J. R. Brent, D. J. Lewis, T. Lorenz, E. A. Lewis, N. Savjani, S. J. Haigh, G. Seifert, B. Derby and P. O'Brien, J. Am. Chem. Soc., 2015, 137, 12689.

2 V. Steinmann, R. Jaramillo, K. Hartman, R. Chakraborty, R. E. Brandt, J. R. Poindexter, Y. S. Lee, L. Sun, A. Polizzotti, H. H. Park and R. G. Gordon, Adv. Mater., 2014, 26, 7488.

3 L. C. Gomes and A. Carvalho, Phys. Rev. B: Condens. Matter Mater. Phys., 2015, 92, 085406.

4 M. Patel, A. Chavda, I. Mukhopadhyay, J. Kim and A. Ray, Nanoscale, 2016, 8, 2293.

5 D. I. Bletskan, M. M. Bletskan and K. E. Glukhov, J. Solid State Chem., 2017, 245, 34.

6 P. Sinsermsuksakul, K. Hartman, S. B. Kim, J. Heo, L. Sun, H. H. Park, R. Chakraborty, T. Buonassisi and R. G. Gordon, Appl. Phys. Lett., 2013, 102, 053901.

7 C. Xin, J. X. Zheng, Y. T. Su, S. K. Li, B. K. Zhang, Y. C. Feng and F. Pan, J. Phys. Chem. C, 2016, 120, 22663.

8 K. T. R. Reddy, N. K. Reddy and R. W. Miles, Sol. Energy Mater. Sol. Cells, 2006, 90, 3041.

9 R. Eymard and A. Otto, Phys. Rev. B: Solid State, 1977, 16, 1616.
10 L. Makinistian and E. A. Albanesi, Phys. Status Solidi B, 2009, 246, 183.

11 B. Ghosh, M. Das, P. Banerjee and S. Das, Semicond. Sci. Technol., 2009, 24, 025024.

12 K. T. Ramakrishna Reddy, N. Koteswara Reddy and R. W. Miles, Sol. Energy Mater. Sol. Cells, 2006, 90, 3041.

13 C. Xia, J. Du, W. Xiong, Y. Jia, Z. Wei and J. Li, J. Mater. Chem. A, 2017, 5, 13400.

14 M. Patel, H. S. Kim and J. Kim, Nanoscale, 2017, DOI: 10.1039/c7nr03370b.

15 M. Ristov, G. Sinadinovski, M. Mitreski and M. Ristova, Sol. Energy Mater. Sol. Cells, 2001, 69, 17.

16 X. Zhou, L. Gan, Q. Zhang, X. Xiong, H. Li, Z. Zhong, J. Han and T. Zhai, J. Mater. Chem. C, 2016, 4, 2111.

17 T. Rath, L. Gury, I. Sanchez-Molina, L. Martinez and S. A. Haque, Chem. Commun., 2015, 51, 10198.

18 F. Lu, J. Yang, R. Li, N. Huo, Y. Li, Z. Wei and J. Li, J. Mater. Chem. C, 2015, 3, 1397.

19 S. Sucharitakul, U. R. Kumar, R. Sankar, F. C. Chou, Y. T. Chen, C. H. Wang, C. He, R. He and X. P. A. Gao, Nanoscale, 2016, 8, 19050.

20 Z. Tian, C. L. Guo, M. X. Zhao, R. R. Li and J. M. Xue, ACS Nano, 2017, 11, 2219.

21 R. Guo, X. Wang, Y. Kuang and B. Huang, Phys. Rev. B: Condens. Matter Mater. Phys., 2015, 92, 115202.

22 Asfandiyar, T. R. Wei, Z. Li, F. H. Sun, Y. Pan, C. F. Wu, M. U. Farooq, H. Tang, F. Li, B. Li and J. F. Li, Sci. Rep., 2017, 7, 43262.

23 L. Medrano Sandonas, D. Teich, R. Gutierrez, T. Lorenz, A. Pecchia, G. Seifert and G. Cuniberti, J. Phys. Chem. C, 2016, 120, 18841.

24 R. Fei, W. Li, J. Li and L. Yang, Appl. Phys. Lett., 2015, 107, 173104.

25 J. Xia, X. Z. Li, X. Huang, N. N. Mao, D. D. Zhu, L. Wang, H. Xu and X. M. Meng, Nanoscale, 2016, 8, 2063.

26 R. E. Banai, L. A. Burton, S. G. Choi, F. Hofherr, T. Sorgenfrei, A. Walsh, B. To, A. Cröll and J. R. S. Brownson, J. Appl. Phys., 2014, 116, 013511.

27 J. Xia, D. Zhu, X. Li, L. Wang, L. Tian, J. Li, J. Wang, X. Huang and X.-M. Meng, Adv. Funct. Mater., 2016, 26, 4673.

28 Z. Zhang, J. Yang, K. Zhang, S. Chen, F. Mei and G. Shen, J. Mater. Chem. C, 2017, DOI: 10.1039/c7tc02865b.

29 C. J. Murphy, T. K. Sau, A. M. Gole, C. J. Orendorff, J. Gao, L. Gou, S. E. Hunyadi and T. Li, J. Phys. Chem. B, 2005, 109, 13857.

30 S. D. Hudson and G. Chumanov, J. Phys. Chem. C, 2008, 112, 19866.

31 J. Wu, N. Mao, L. Xie, H. Xu and J. Zhang, Angew. Chem,. Int. Ed., 2015, 54, 2366.

32 X. Wang, A. M. Jones, K. L. Seyler, V. Tran, Y. Jia, H. Zhao, H. Wang, L. Yang, X. Xu and F. Xia, Nat. Nanotechnol., 2015, 10, 517.

33 H. B. Ribeiro, M. A. Pimenta, C. J. S. de Matos, R. L. Moreira, A. S. Rodin, J. D. Zapata, E. A. T. de Souza and A. H. C. Neto, ACS Nano, 2015, 9, 4270.

34 J. Kim, J. U. Lee, J. Lee, H. J. Park, Z. Lee, C. Lee and H. Cheong, Nanoscale, 2015, 7, 18708. 
35 X. Ling, S. Huang, E. H. Hasdeo, L. Liang, W. M. Parkin, Y. Tatsumi, A. R. Nugraha, A. A. Puretzky, P. M. Das, B. G. Sumpter, D. B. Geohegan, J. Kong, R. Saito, M. Drndic, V. Meunier and M. S. Dresselhaus, Nano Lett., 2016, 16, 2260.

36 A.-L. Phaneuf-L'Heureux, A. Favron, J.-F. Germain, P. Lavoie, P. Desjardins, R. Leonelli, R. Martel and S. Francoeur, Nano Lett., 2016, 16, 7761.

37 W. Lu, X. Ma, Z. Fei, J. Zhou, Z. Zhang, C. Jin and Z. Zhang, Appl. Phys. Lett., 2015, 107, 021906.
38 W. Luo, Q. Song, G. Zhou, D. Tuschel and G. Xia, arXiv, 1610.03382, 2016.

39 P. M. NikoliC, L. j. Iljkovic, P. Mihajlovic and B. LavrenEic, J. Phys. C: Solid State Phys., 1977, 10, L289.

40 R. C. Sharma and Y. A. Chang, J. Phase Equilib., 1986, 7, 269.

41 H. R. Chandrasekhar, R. G. Humphreys, U. Zwick and M. Cardona, Phys. Rev. B: Solid State, 1977, 15, 2177.

42 A. C. Albrecht, J. Chem. Phys., 1961, 34, 1476.

43 G. A. Tritsaris, B. D. Malone and E. Kaxiras, J. Appl. Phys., 2013, 113, 233507. 